Radial and Nonradial Pulsations as Probes of Stellar Physics

ASP Conference Series, Vol. 259, 2002

C. Aerts, T.R. Bedding, \& J. Christensen-Dalsgaard, eds.

\title{
Quasi-Periodic Oscillations in Accretion Discs
}

\author{
Brian Warner, Patrick A. Woudt \\ Department of Astronomy, University of Cape Town, Rondebosch 7700, \\ South Africa
}

Quasi-Periodic Oscillations (QPOs) in Cataclysmic Variable stars (CVs) with periods in the range $100-2000 \mathrm{~s}$ have been known for 25 years (see Chapter 8 of Warner, 1995a). We distinguish here between QPOs and Dwarf Nova Oscillations (DNOs) - the latter being of shorter period $(5-50 \mathrm{~s})$ and greater coherence. QPOs appear as brightness modulations and typically have quite short coherence lengths (a few cycles).

QPOs are largely associated with discs transferring mass at a high rate i.e., dwarf novae during outburst or nova-like variables; but the QPOs are not always present and many systems have not shown QPOs at all.

Generally it is thought that QPOs result from non-radial oscillations of the accretion discs surrounding the white dwarf component in CVs (Carroll et al., 1985; Collins et al., 2000). They could, e.g., be quite coherent oscillations of individual annuli in a disc, each with a different period. Alternatively, a particular oscillation mode may be excited for only a few cycles and then be reexcited at an arbitrary phase. Most theoretical proposals have sought to make the QPOs self-luminous pulsations of the disc, in analogy with the kappa or epsilon processes of stellar pulsation.

The left panel of Fig. 1 shows the light curve of the dwarf nova VW Hyi that we obtained on 5 Feb 2000 as the star faded through the final stage of a normal outburst. The large humps are orbital modulation, there are DNOs present throughout the run (see inset) but there are also QPOs, seen as slower and irregular humps with a peak-to-peak range of $\sim 0.1 \mathrm{mag}$ and a mean period that increased from $\sim 400 \mathrm{~s}$ to $\sim 600 \mathrm{~s}$ during the observation.
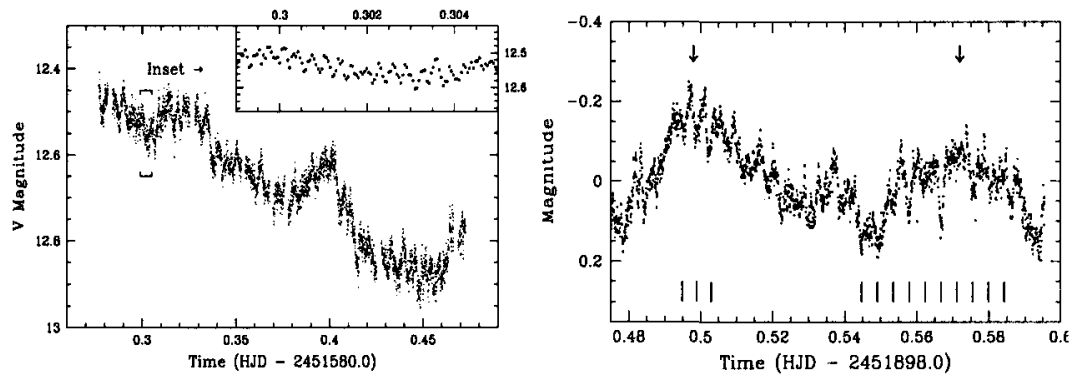

Figure 1. Light curves of VW Hyi. For details see text.

QPOs are often not quasi-sinusoidal modulations - their minima are sometimes dips that lie below the general envelope of the general background and 
flickering, as though caused by obscuration (see right panel of Fig. 1 where two groups of recurrent dips are marked by vertical bars, and arrows point to orbital humps). This leads us to the following interpretation of QPOs.

In a general investigation of the propagation of waves in an accretion disc Lubow \& Pringle (1993) concluded that the $m=1$ g-mode appears to provide the best propagational properties near the disc centre. Together with the above observations this suggests to us that the QPOs are caused by prograde travelling waves in the inner disc. These may be self-luminous, but their principal reason for generating brightness modulations is that they intercept radiation from the luminous white dwarf (heated by accretion). When on the far side (superior conjunction) the wall is irradiated by UV flux from the primary and reprocesses some of it in our direction; at inferior conjunction it does not produce that extra optical flux, and may indeed partially obscure the primary.

There is another type of observation that supports this model. In OY Car (Marsh \& Horne, 1998), in V2051 Oph (Steeghs et al., 2001), and in our observations of VW Hyi examples have been seen of double DNOs - the simultaneous presence of two DNOs at slightly different periods. In VW Hyi we find that the difference frequency of the DNOs is equal to the frequency of the QPO present at the same time. Our interpretation is that the rotating beam from the primary (which generates the DNOs) is irradiating a prograde travelling wall.

We have reanalysed light curves of WZ Sge, where DNOs at $27.868 \mathrm{~s}$ and $28.952 \mathrm{~s}$ are often present. We have for the first time detected a strong modulation at the beat period (742 s) of these DNOs, which is similar to what is seen in VW Hyi and gives support to a DNO model for the WZ Sge modulations.

A remaining question is why not all CV discs show QPOs. We have proposed a mechanism whereby winding up and breaking of magnetic field lines joining the primary and the inner disc can excite a travelling wave in the inner disc. The parameter that varies from system to system and determines whether QPOs will appear may therefore be the field strength of the primary. This, and the magnetic accretion model for the production of DNOs (Warner, 1995b), suggests that even 'non-magnetic' CVs may have weak fields (less than about $10^{5}$ Gauss).

\section{References}

Carroll, B.W., Cabot, W., McDermott, P.N., Savedoff, M.P., \& van Horn, H.M. 1985, ApJ, 296, 529

Collins, T.J.B., Helfer, H.L., \& van Horn, H.M. 2000, ApJ, 534, 934

Lubow, S.H., \& Pringle, J.E. 1993, ApJ, 409, 360

Marsh, T.R., \& Horne, K. 1998, ApJ, 299, 92

Steeghs, D., O'Brien, K., Horne, K., Gomer, R., \& Oke, J.B. 2001, MNRAS, in press

Warner, B. 1995a, Cataclysmic Variable Stars (Cambridge University Press: Cambridge)

Warner, B. 1995b, in ASP Conf. Ser., Vol. 85, Cape Workshop on Magnetic Variables, eds. D.A.H. Buckley \& B. Warner, (San Francisco: ASP), 343 


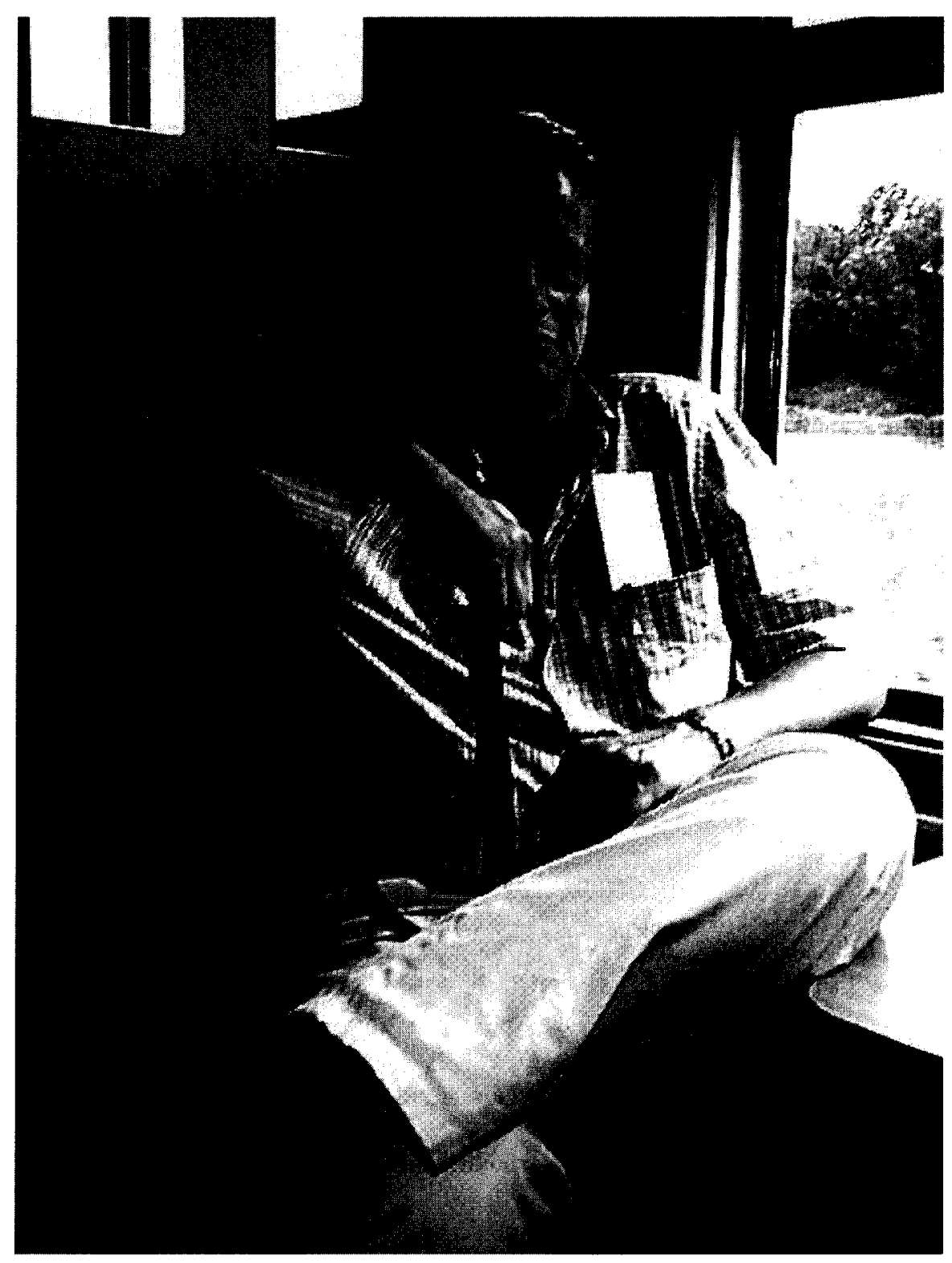

One happy man (Dietrich Baade) returning home on the same train after the conference. 